\title{
Formación profesional docente y desempeño laboral de los docentes
}

\section{Teacher training and professional performance of teachers}

1 Jhonatan Guillermo Fauta Aulestia

https://orcid.org/0000-0002-9133-3649

Universidad Técnica de Ambato, Facultad de Ciencias Humanas y de la Educación,

Carrera de Psicopedagogía. Ambato, Ecuador.

jfauta5481@uta.edu.ec

2 Karen Vanessa Palomo Rivera

https://orcid.org/0000-0003-0981-7976

Universidad Técnica de Ambato, Facultad de Ciencias Humanas y de la Educación,

Carrera de Psicopedagogía. Ambato, Ecuador.

kaplomo5611@uta.edu.ec

3 Carmen del Rocío Núñez López $\quad$ (iD) https://orcid.org/0000-0002-4300-7048.

Universidad Técnica de Ambato, Facultad de Ciencias de la Educación, Carrera de

Psicopedagogía. Ambato, Ecuador.

carmennuñezl@uta.edu.ec

4 Verónica del Carmen Llerena Poveda (iD) https://orcid.org/0000-0002-5962-7962

Universidad Técnica de Ambato, Facultad de Ciencias de la Educación, Carrera de

Psicopedagogía. Ambato, Ecuador.

veronicadllerena@uta.edu.ec

Artículo de Investigación Científica y Tecnológica

Enviado: 24/12/2021

Revisado: $29 / 12 / 2021$

Aceptado: $12 / 01 / 2022$

Publicado:08/03/2023

DOI: https://doi.org/10.33262/concienciadigital.v6i1.4.2011

Fauta Aulestia, J. G., Palomo Rivera, K. V., Núñez López, C. del R., \& Llerena Poveda, V. del C. (2023). Formación profesional docente y desempeño laboral de los docentes . ConcienciaDigital, 6(1.4), 506-523. https://doi.org/10.33262/concienciadigital.v6i1.4.2011

CONCIENCIA DIGITAL, es una Revista Multidisciplinar, Trimestral, que se publicará en soporte electrónico tiene como misión contribuir a la formación de profesionales competentes con visión humanística y crítica que sean capaces de exponer sus resultados investigativos y científicos en la misma medida que se promueva mediante su intervención cambios positivos en la sociedad. https://concienciadigital.org .

La revista es editada por la Editorial Ciencia Digital (Editorial de prestigio registrada en la Cámara Ecuatoriana de Libro con No de Afiliación 663) www.celibro.org.ec 


\section{Palabras} claves: formación docente, desempeño laboral, saber pedagógico, competencias del docente, capacitación.

Keywords: teacher training, job performance, pedagogical knowledge, teacher skills,

\section{Resumen}

Introducción: La formación profesional y desempeño docente, son factores que caracteriza el proceso de enseñanza aprendizaje en los distintos niveles de educación, está asociada a la emergencia de generar nuevas formas de conocimiento en el proceso de la ciencia, Hay aspectos que no nos permite identificar errores que fácilmente pasan desapercibidos y por lo tanto son ignorados, en consecuencia, no se logra mejorar el nivel educativo de la institución y se da el bajo rendimiento académico de los alumnos, se presume que una de las principales causas de ello es provocado por un bajo nivel o conocimiento del desempeño docente, estilos de crianza no acorde para los niños entre otros, Objetivo: Analizar la importancia y relación de la formación y desempeño docente acciones que cumple dentro de la educación. Metodología: Para esta investigación hemos considerado el enfoque mixto porque nos permite combinar dos variables y poder utilizar instrumentos de evaluación IACD que contiene 3 dimensiones, la población fue de 50 personas, es una investigación tipo descriptiva y correlacional porque se encarga de definir y detallar los distintos componentes educativos, en la unidad educativa Pujilí. Resultados: Los resultados obtenidos demuestran que encuestados nos indica que existe un nivel de correlación alta es decir que aun nivel mayor de formación profesional docente mayor será el nivel de desempeño docente. Con una prueba de normalidad arroja el valor de $\mathrm{P}$ es mayor que 0.05 por ello señala que existe una dispersión anormal dentro de los datos de la investigación. Conclusiones: mediante el análisis de ambas variables se concluye que, si bien los docentes presentan un excelente auto concepto en relación con sus competencias como docentes es necesario no dejar a un lado las capacitaciones o las actividades extracurriculares que pueden mejorar el perfil dinamizador del docente y no permitir el conformismo con el conocimiento nuevo que adquieren.

\section{Abstract}

Introduction: professional training and teaching performance are factors that characterize the process of teaching and learning at different levels of education, which is associated with the emergence of generating new forms of knowledge in the process of science. There are aspects that do not allow us to identify errors that 
training. easily go unnoticed and therefore are ignored, as result, the educational level of the institution is not improved and there is the low academic performance of students, it is presumed that one of the main causes of this is caused by a low level or knowledge of teaching performance, parenting styles not appropriate for children among others, Objective: To analyze the importance and relationship of teacher training and performance actions carried out within education, Methodology: For this research we have considered the mixed approach because it allows us to combine two variables and be able to use IACD assessment instruments that contain 3 dimensions, the population was 50 people, it is a descriptive and correlational type research because it is responsible for defining and detailing the different components educational, in the educational unit Pujilí. Results: The results obtained show that those surveyed indicate that there is a high level of correlation, that the higher the level of professional teacher training, the higher the level of teacher performance. With a normality test, the value of $\mathrm{P}$ is greater than 0.05 , which is why it indicates that there is an abnormal dispersion within the research data. Conclusions: through the analysis of both variables, it is concluded that, although teachers have an excellent self-concept in relation to their skills as teachers, they are not.

\section{Introducción}

\section{Formación profesional docente}

Según Suco (2020) la formación docente son factores que caracteriza el proceso de enseñanza aprendizaje en los distintos niveles de educación, son responsabilidades que adquieren el profesorado donde su comportamiento es moldeable para integrase y acoplar a las reglas y normas institucionales. Los docentes son un eje fundamental para un correcto conocimiento, por lo que la formación inicial y permanente, el proceso de formación, incorporación y condiciones de trabajo y de más aspectos determinan el impacto que tienen sobre el aprendizaje de todos los dicentes dentro de la comunidad educativa.

El Ministerio de educación [MINEDUC], (2020), y otros organismos consideran que la formación docente debe ser un proceso continuo por ello se implementaran un plan de formación para actualización de conocimiento que favorece positivamente a la educación y brindar un correcto aprendizaje de calidad a los dicentes, la formación profesional docente 
es considera como un mecanismo permanente de autorreflexión sobre la práctica educativa del docente, además, nos permite modificar, transformar y fortalecer habilidades, competencias y destrezas transversales, disciplinares y socioemocionales de los profesionales educativos.

El desarrollo del docente se puede dar de diferentes perspectivas ya sea individual o institucional, porque el docente puede determinar cuáles son sus debilidades y empezar a trabajar y mejorar, es importante que la institución este en constate seguimientos a los maestros para saber que profesionales se encuentran a cargo de los alumnos y que temas le están enseñando. Por otro lado, el aspecto institucional hace referencia a los requisitos que él se tiene que cumplir como docente para poder contribuir y trabajar dentro de una institución educativa. La formación docente se vincula con el desatollo del aprendizaje, la metodología, la ciencia y la indagación de nuevos cuestionamientos para fortalecer la educación de manera global.

Factores del desarrollo profesional docente

Se determina aspectos importantes para un mejor desenvolvimiento del personal docente dentro del ámbito educativo los mis os que son (Gestión Educativa, 2016).

\section{Tabla 1}

\section{Factores del desarrollo docente}

Formación continua Es importante que el personal docente se encuentre en constante aprendizaje para poder impartir mayor conocimiento a los estudiantes.

Reciclaje Hace referencia a que el docente debe adaptarse al contenido que se a ser visto durante la clase, y el mensaje sea claro para los alumnos.

Profesionalización No hace referencia al título que tenemos, si no a que tan buenos profesionales somos al momento de impartir clases a sus estudiantes acorde a sus capacidades y necesidades que necesitan ser identificadas, para cambiar de metodología.

Crecimiento profesional Aquí tiene que ver mucho la vocación que tienen el profesional paracon sus estudiantes, por consiguiente, a ello se ve evidenciada la empatía, relación interpersonal, alumno-docente.

Fuente: Navarro (2018)

La formación profesional docente se lo relaciona con la búsqueda de buscar formas de concebir el conocimiento y los procesos del saber, en general, debe enmarcase cada una de las etapas de formación docente en el aprendizaje y bienestar estudiantil ya que son agentes mediadores dentro del proceso, son motivadores e impulsadores para la resolución de conflictos. 
Fases de la formación permanente del docente

Tabla 2

Fases de formación docente

Evaluación Se encentra inmersa la retroalimentación para la mejora del proceso enseñanza aprendizaje.

Ejecución

Se implementa itinerarios para facilitar la formación individual del docente.

Diagnostico

Se realiza un análisis tanto de la evaluación que se realizan al docente como a los estudiantes.

Reflexión

Es un espacio para la reflexión, comprensión, indagación de las necesidades y problemas que puede existir dentro de la institución, para poder crear nuevas prácticas educativas.

Planificación

Tomar en cuenta siempre la formación continua con un enfoque de inclusión y calidad y determinación de asignaturas o actividades acorde al perfil profesional.

Fuente: Navarro (2018)

\section{Competencias de docente}

Se menciona algunas de las competencias que el día de hoy se debe cumplir para un buen aprendizaje de los estudiantes (Navarro, 2018).

Dominar los saberes a enseñar: para logar que los alumnos capten la clase hay que dominar el tema a abordar e incentivar a que se interesen del tema y no se quede ahí y ellos mismo se brinden el autoconocimiento necesario en su tiempo libre, con esto se lograra a que vayan más allá de lo que se pretende enseñar, para ello es importante que se priorice y jerarquizar los contenidos de tal manera que favorezca al aprendizaje.

Actuar acorde a las características y necesidades que presente el estudiante: de toma en cuenta cada una de las maneras de aprender del alumno, como se puede ver la enseñanza no es colectiva sino individual ya que como docenes tienen que estudiar a cada uno de sus estudiantes para poder elegir la metodología adecuada que beneficie a todos, para ellos podemos hacer uso de los estilos de aprendizaje los cuales son, estilos de aprendizaje visual, kinestésico y auditivo.

Dirigir y gestionar la enseñanza: los docentes tendrán que plantear los objetivos por consiguiente verificar si los recursos didácticos son los adecuadas para el tema, se manifestara al maestro que tome la cuenta el tiempo que le va a llevar, el espacio y si las estrategias a implementar son o no las adecuadas. Dentro de ello tenemos la evaluación la 
cual debemos considerarla como procesos de retroalimentación o feedback después de cada tema para mayor comprensión por parte de los dicentes en donde se evidenciará si el alumno está o no aprendiendo, por ello se realiza un seguimiento a cada estudiante para avisar a las autoridades y padres de familia de su rendimiento.

Intervenir en la dinámica trabajo grupal: dar a conocer lo fundamental de reforzar y hacer uso de las habilidades sociales para una buena relación interpersonal con sus pares y demás personas, y la convivencia sea agradable.

Propio proceso formativo: como lo hemos venido mencionando anteriormente es responsabilidad de cada uno y de la institución estar en contante formación y actualización de conocimientos por el bienestar de los estudiantes y de la comunidad educativa.

\section{El saber pedagógico}

Son los conocimientos construidos por los docentes ya sean los valores, actitudes e ideologías dentro del contexto cultural, social y educativo, el saber nos da una amplia visión considerando la práctica, forma de trabajo y la reflexión, aquí la práctica levara a unacotidianidad para generar un saber acumulado dentro de la docencia. El saber está conformado por elementos que nos hacen fácil de identificar, como son los distintos objetos para la práctica, los conocimientos que se adquieren y se ponen en evidencia.

El saber pedagógico es cuando un docente pone en evidencia un tema para abordar dentro de la clase, lo simplifican, lo preparan, es decir buscan la manera, el lugar más idóneo para que el tema llegue a los estudiantes de manera fácil, practica y eficiente y gracias a ello se puede evidenciar la limitación que existe dentro del aula (Amaya, 2016).

\section{Formación por competencias laborales}

Es comprendida como el proceso de enseñanza aprendizaje dirigida para las personas a que adquieran habilidades y destreza y conocimiento para lo cual se empleara nuevos procedimientos y modificación de conducta si así lo necesita para mejorar su desempeño, esto se lo debe hacer de manera continua tanto dentro del ámbito laborar, estudiantil, y profesional para que no exista rebasamiento por nuevo personal u otras cuestiones $\mathrm{y}$ necesitan readaptarse a las nuevas tendencias, parte del aprendizaje significativo y formación de la personas como parte principal del proyecto pedagógico. Para ellos es necesario hacer uso de técnicas didácticas que refuercen el conocimiento y permita un buen desenvolvimiento en los distintos contextos laborales (Martínez, 2018).

\section{Desempeño laboral docente}

El desempeño laboral es considerado rendimiento que presenta un maestro frente a determinada labor, de esta manera de determina si es apto o no para estar al frente de un 
determinado grupo de alumnos. Es la acción que desempeña un laborador en este caso el docente en la institución como practica de enseñanza para poder evidenciar su capacidad profesional, su responsabilidad profesional, componentes que sea de gran impacto en el contexto educativo, también tiene responsabilidad por la gestión educativa (Potosí, 2017).

Acorde a lo que mencionan el desempeño laboral docente está conformado por apartados fundamentales como es el rol del docente, capacidad docente, perfil docente, proceso profesional $\mathrm{y}$, es importante mencionar que el ambiente y la práctica de aprendizaje enseñanza que desarrolle el docente Demostrará la importancia que el docente $\mathrm{n}$ en el centro educativo.

Por otra parte, se menciona que el desempeño laboral docente es el eje fundamental en el cual se moviliza el proceso de formación dentro del sistema educativo formal, además se logra identificar errores que fácilmente pasan desapercibidos y no son observados, como consecuencia de ello, no se logra mejorar el nivel educativo de la institución y de allí nace una gran problemática a nivel institucional, se debe tomar las medidas necesarias para que no tenga gran impacto en la comunidad educativa (García, 2017).

\section{Funciones del desempeño docente}

Es un elemento esencial para mantener la trasparencia del sistema educativo, Se conoce el proceso sistemático en el cual la evaluación del desempeño docente toma mayor sentido, ya que se propone que la evaluación del desempeño docente tiene que efectuar y constatar con al menos las siguientes funciones.

- Función de diagnóstico: ayuda a determinar fácilmente que es lo que pasa dentro del contexto educativo y encontrar la solución de cómo podrían abordar y corregir una determinada situación.

- Función instructiva: es una autoevaluación, para la incorporación de nuevos conocimientos para la práctica pedagógica y perfección de los estudiantes en los conocimientos y tener un buen avance.

- Función educativa: su finalidad es transmitir los procesos que se llevan a cabo dentro de la institución, también hace referencia a que no simplemente los maestros dicten su tema, si no que el dicente se adueñe de él y lo interprete.

- Función desarrolladora: hace referencia a los resultados que se presenta cuando existe una evaluación dentro de las aulas son favorables y han sido productivas para todos por los avances logrados, y que sea capaz de afrontar a sus errores cometidos.

Estas con el fin de detectar problemas o diferentes aspectos que puedan limitar al proceso de enseñanza aprendizaje, evidencia que la práctica docente sea optima, y con forme a ello incorporar nuevos medios educativos para ajustar a lo planificado acorde al tema que 
se va a proporcionar durante la clase.

\section{Fines de la evaluación al desempeño docente}

Acorde a lo que menciona el Ministerio de Educación (MINEDUC, 2020), dice que la evaluación a los docentes nos permitirá promover, conocer e implantar nuevas acciones didácticas pedagógicas con el principal fin de la evaluación al desempeño docente que es la de enriquecer la práctica pedagógica para ofrecer un feedback apropiado al personal docente con esto asegurar un aprendizaje valioso para el estudiante.

Por otro lado, la intención principal es determinar cualidades profesionales y autónomas del docente, las mismas que proporcionaran el desarrollo de metodologías y estrategias de formación apropiadas para el docente.

\section{Desempeño docente y rendimiento académico}

El rendimiento académico no depende únicamente del estudiante, si no que el docente tendrá que realizar una evaluación para determinar cuál es su nivel de conocimiento y posterior a ellos utiliza la metodología apropiada para implementar dentro de la clase. Todo dependerá de la trasformación que tenga la institución con un enfoque innovador y docentes comprometidos a un cambio, lo cual nos ayudará en la toma de decisiones, y orientar en la práctica pedagógica (Mariana, 2019).

En la actualidad, el bajo rendimiento académico de los alumnos es provocado por un deficiente nivel del desempeño, conocimiento docente, y falta de vocación. Pero también toma en cuenta diversos aspectos lo que estará provocando este bajo rendimiento, problemas dentro de la familia o los padres están ausentes porque trabajan todo el tiempo, hijos predilectos en fin todo esto conlleva hacer Un análisis de la importancia de visualizar al profesorado como un todo, y aquí dentro del aula de clases desarrolla habilidades como el liderazgo, trabajo de equipo y sus competencias como docente, adicional a todo esto un conocimiento sobre psicología para poder generar reporte y mantener en observación a los alumnos, esto con el fin de que consiga manejar de manera favorable al grupo en cuestión.

\section{Metodología}

La presente investigación lleva una modalidad de tipo mixto, ya que nos ayuda a combinar dos enfoques y la utilización de distintas técnicas para la obtención de datos, que se obtuvieron en base a los Instrumentos validados, los cuales se utilizaron para obtener la información de las dos variables sobre los docentes de la Unidad Educativa Provincia De Cotopaxi.

Es de tipo descriptiva y correlacional ya que detalla y defina los fenómenos educativos en el contexto circunstancial, espacial y temporal. Nos permite explicar los elementos que dan un diagnóstico de la situación estudiada, En consecuencia, el nivel correlacional detalla 
en qué medida la formación profesional docente acepta el desempeño laboral, generando un análisis más detallado de la relación entre variables de estudio, basada en revisión de fuentes bibliográficas confiables (Sampieri, 2018).

El estudio pretende identificar si la formación docente, y el desempeño laboral es la adecuada para proceso de enseñanza aprendizaje, Los datos tomados en la Unidad Educativa Provincia de Cotopaxi del cantón Pujilí nos permiten identificar el nivel de las variables estudiadas, para ello se tomara en cuenta los instrumentos de autoevaluación de competencias docentes en la formación docente (IACD) cuestionario que se lograra determinar y conocer del nivel de formación docente, de la misma manera la aplicación de la evaluación del desempeño docente, por parte de los alumnos que medirá y calificara de manera clara y concisa el desempeño docente de cada uno de los docentes de la institución educativa (Ornelas, 2016).

A partir de la información de desarrollo se el análisis descriptivo de la recolección de los datos de cada una de las variables la formación docente y el desempeño laborar, la cual se realizó de manera virtual.

\section{Resultados}

Análisis e interpretación del Instrumento de Autoevaluación de Competencias Docentes en la Formación Profesional (IACD).

Informe de la autoevaluación aplicado a docentes de la Unidad Educativa Provincia de Cotopaxi del bloque 1.

Calificación final del instrumento de autoevaluación de competencias docentes en la formación profesional (IACD).

\section{Tabla 3}

Calificación final del instrumento IACD

\begin{tabular}{lll}
\hline \multicolumn{1}{c}{ Frecuencia } & Muestra & \multicolumn{1}{c}{ Porcentaje } \\
\hline Correcto & 45 & $90 \%$ \\
Aceptable & 5 & $10 \%$ \\
Mejorable & 0 & $0 \%$ \\
TOTAL & 50 & $100 \%$
\end{tabular}

Fuente: Estudio en el contexto de la Unidad

Educativa Provincia de Cotopaxi. 


\section{Figura 1}

Resultado final del instrumento IACD

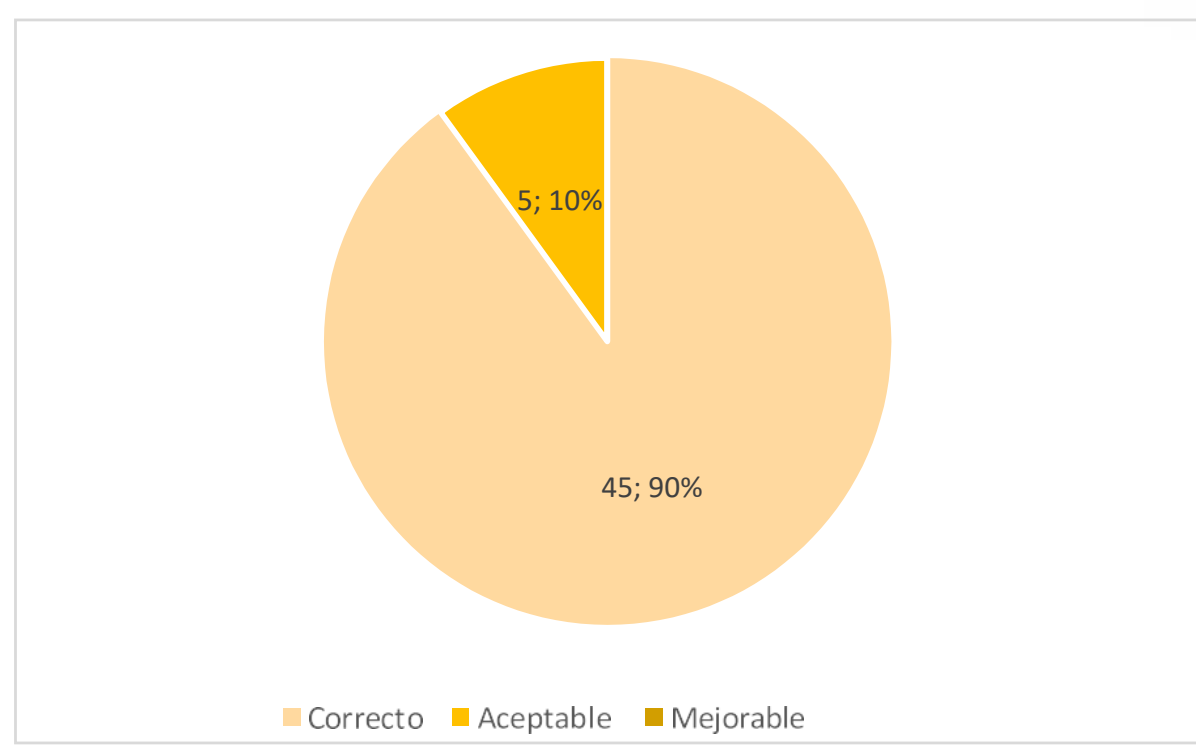

Fuente: Estudio en el contexto de la Unidad Educativa Provincia de Cotopaxi

Nota: Datos obtenidos de la aplicación del instrumento IACD en Google forms.

Se determina que e1 $90 \%$ de los encuestados se encuentran en un nivel correcto en relación con las 3 áreas o dimensiones que abarca el instrumento de autoevaluación de competencias docentes. El 10\% restante están en un nivel aceptable con relación a cada una de las competencias que se evaluó en el instrumento.

En conclusión, se demuestra que un alto porcentaje de la población docente percibe que su labor como maestro o profesional es correcta y aceptable dentro de los parámetros que se consideraron pertinentes al momento de seleccionar el instrumento de evaluación, esto puede verse reflejado en el cumplimiento de objetivos académicos por parte de los estudiantes.

Análisis e interpretación del instrumento de evaluación del desempeño docente, por parte de los alumnos.

Informe de la autoevaluación aplicado a estudiantes de tercero de bachillerato de la Unidad Educativa Provincia de Cotopaxi del cantón Pujilí.

Calificación final del instrumento de evaluación del desempeño docente, por parte de los alumnos. 


\section{Tabla 4}

Calificación final de la evaluación al desempeño docente por parte de los alumnos

\begin{tabular}{lll}
\hline Frecuencia & Muestra & Porcentaje \\
\hline EXCELENTE & 30 & $60 \%$ \\
MUY BUENO & 19 & $38 \%$ \\
BUENO & 1 & $2 \%$ \\
REGULAR & 0 & $0 \%$ \\
PÉSIMO & 0 & $0 \%$ \\
TOTAL & 50 & $100 \%$
\end{tabular}

Fuente: Estudio en el contexto de la Unidad

Educativa Provincia de Cotopaxi

\section{Figura 2}

\section{Calificación final del instrumento de evaluación del desempeño docente}

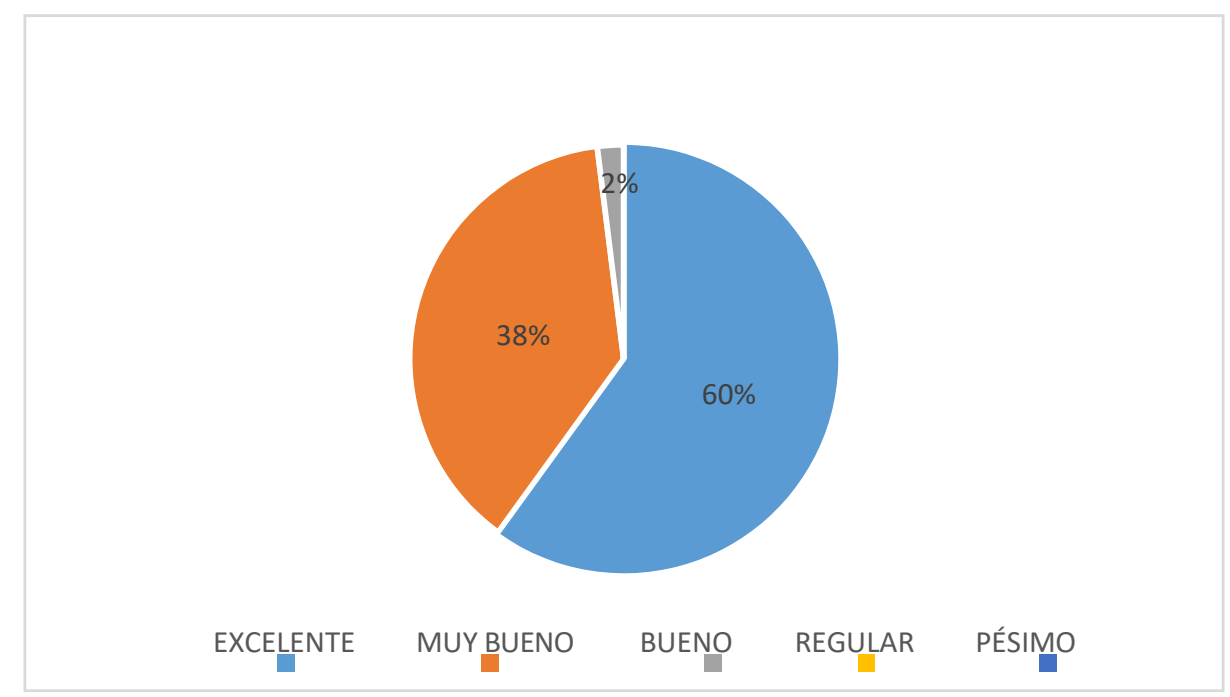

Fuente: Estudio en el contexto de la Unidad Educativa Provincia de Cotopaxi

Nota: Datos obtenidos de la aplicación del instrumento en Google forms.

La evaluación del desempeño laboral por parte de los estudiantes Arrojó que el valor que mayor frecuencia tuvo es la de excelente con lo cual logramos comprender y entender que los docentes de la Unidad Educativa Provincia de Cotopaxi presentan un excelente nivel del desempeño profesional en sus labores docente, Por otra parte con una frecuencia de 19 el valor de muy bueno dentro de las evaluaciones del desempeño docente no saben entender que existe un correcto manejo de las distintas competencias y habilidades que son necesarias para la práctica educativa por parte de los docentes por 
último existe únicamente una frecuencia de 1 en relación a un buen desempeño docente que si bien no presenta mayor dificultad si existe la necesidad de mejorarlo con el fin de incrementar las posibilidades de aprendizaje de los estudiantes.

La evolución del personal docente es de gran utilidad para él lograr identificar las habilidades y las áreas fuertes de los docentes Asimismo las áreas débiles y carencias en la práctica educativa, por lo cual este instrumento nos ha dado a entender de que gran parte del personal docente maneja de manera adecuada muchos elementos del currículo para ofrecer una enseñanza de calidad, sin embargo, se puede mejorar cada 1 de los elementos curriculares principalmente las competencias que ejerce el docente como es el proceso de enseñanza aprendizaje ya que es en esta dimensión en la cual se encontró mayor dispersión de la frecuencia y mayor dificultad en la evaluación del desempeño docente.

\section{Prueba de Normalidad}

La prueba de normalidad es un método utilizado para para indicar si se debe rechazar o no se debe rechazar la hipótesis nula, además es utilizada frecuentemente para indicar el tipo de prueba estadística de correlación qué se debe aplicar, esto dependiendo de la normalidad de la distribución de los datos en relación de la frecuencia y la población, por esta razón la prueba de normalidad es una de las mejores herramientas para evaluar la normalidad de los datos en una investigación.

La prueba de la normalidad se logra interpretar a realizado el valor que arroja $\mathrm{P}$ ya que si el valor de $\mathrm{P}$ es mayor que 0.05 estamos hablando de una dispersión normal de los datos estadísticos, por otra parte, si el valor de $\mathrm{P}$ es menor a 0.05 no señala que existe una dispersión anormal dentro de los datos de la investigación.

\section{Prueba de normalidad de Anderson-Darling}

Se caracteriza por determinar si un conjunto de datos proviene de una distribución especifica, para lo cual se utiliza la siguiente formula:

$$
A D=-n-\frac{1}{n} \sum_{i=1}^{n}(2 i-1)\left[\ln F\left(X_{i}\right)+\ln \left(1-F\left(X_{n-i+1}\right)\right)\right]
$$

$\mathrm{n}=$ tamaño de la muestra

$\mathrm{F}(\mathrm{X})$ = función de distribución acumulativa para la distribución especificada.

$\mathrm{i}=$ la i- ésima muestra cuando los datos se ordenan en orden ascendente.

A menudo verá esta estadística llamada $A^{2}$. 
Prueba de normalidad de la variable formación profesional docente

\section{Figura 3}

\section{Gráfico de probabilidad de formación docente}

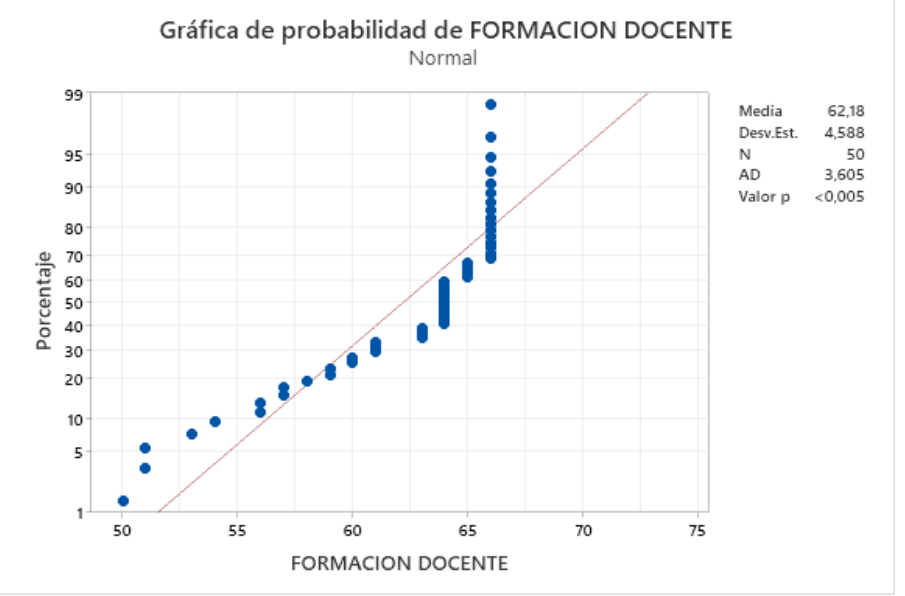

Fuente: Estudio en el contexto de la Unidad Educativa Provincia de Cotopaxi

Nota: Datos de la aplicación de la prueba normalidad de Anderson Darling en Minitab

En concordancia con lo mencionado anteriormente el valor de $\mathrm{P}$ de la prueba de normalidad de Anderson Darling arroja que la variable de formación profesional docente cuenta con un valor de $\mathrm{P}$ de 0.005 por lo cual se concluye que los datos son de naturaleza no normal.

Prueba de normalidad de la variable desempeño laboral docente

\section{Figura 4}

Gráfico de probabilidad de desempeño docente

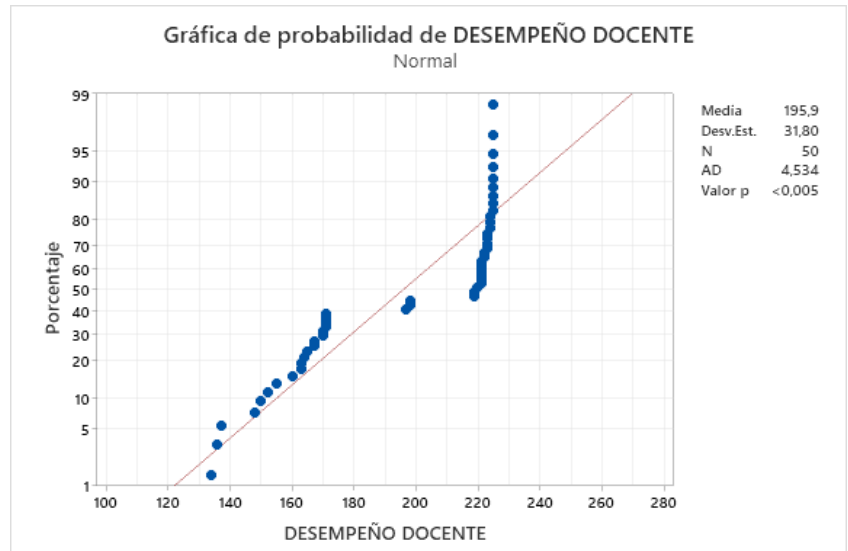

Fuente: Estudio en el contexto de la Unidad Educativa Provincia de Cotopaxi

Nota: Datos de la aplicación de la prueba normalidad de Anderson Darling en Minitab 
La gráfica que nos arroja de la prueba de normalidad de Anderson Darling en relación con la variable desempeño laboral docente nos muestra que los datos no se encuentran agrupados, Por otra parte, el valor de $\mathrm{P}$ es de 0.005 con lo cual se concluye que los datos son de naturaleza no normal.

Resultado final de la prueba de normalidad

Tabla 5

Resultado final de la prueba de normalidad

\begin{tabular}{l|l|l|l|l}
\hline VARIABLE & MEDIA & $\begin{array}{l}\text { VALOR } \\
\text { DE P }\end{array}$ & $\begin{array}{l}\text { NATURALEZA DE LOS } \\
\text { DATOS }\end{array}$ & $\begin{array}{l}\text { Estadístico de } \\
\text { correlación }\end{array}$ \\
\hline $\begin{array}{l}\text { FORMACIÓN } \\
\text { PROFESIONAL } \\
\text { DOCENTE }\end{array}$ & 62.18 & $<0.005$ & $\begin{array}{l}\text { Los datos no } \\
\text { siguen la } \\
\text { distribución especificada }\end{array}$ & Pearson. \\
\hline $\begin{array}{l}\text { DESEMPEÑO } \\
\text { LABORAL DOCENTE }\end{array}$ & 195.9 & $<0.005$ & $\begin{array}{l}\text { Los datos no } \\
\text { siguen la }\end{array}$ & Pearson. \\
& & distribución especificada
\end{tabular}

Fuente: Estudio en el contexto de la Unidad Educativa Provincia de Cotopaxi

\section{Correlación de Pearson}

La correlación se lo realizará mediante la prueba de Pearson ya que al contar con datos anormales es necesario incorporar este tipo de prueba, con la incorporación de esta prueba estadística pretende establecer el tipo de relación que existe entre las dos variables, a continuación, se explica de manera detallada el cómo se interpreta el valor arrojado por la prueba de correlación de Pearson.

\section{Tabla 6}

Valores de la correlación de Pearson

\begin{tabular}{ll} 
VALOR & EQUIVALENCIA \\
\hline $\mathrm{r}=1$ & Correlación perfecta \\
\hline $0.8<\mathrm{r}<1$ & Correlación muy alta \\
\hline $0.6<\mathrm{r}<0.8$ & Correlación alta \\
\hline $0.4<\mathrm{r}<0.6$ & Correlación moderada \\
$0.2<\mathrm{r}<0.4$ & Correlación baja \\
\hline $0<\mathrm{r}<0.2$ & Correlación muy baja \\
\hline $\mathrm{r}=0$ & Correlación nula
\end{tabular}

Fuente: Estudio en el contexto de la Unidad Educativa Provincia de Cotopaxi. 
Correlación formación profesional docente y desempeño laboral

\section{Figura 5}

Gráfica de correlación de formación y desempeño docentes

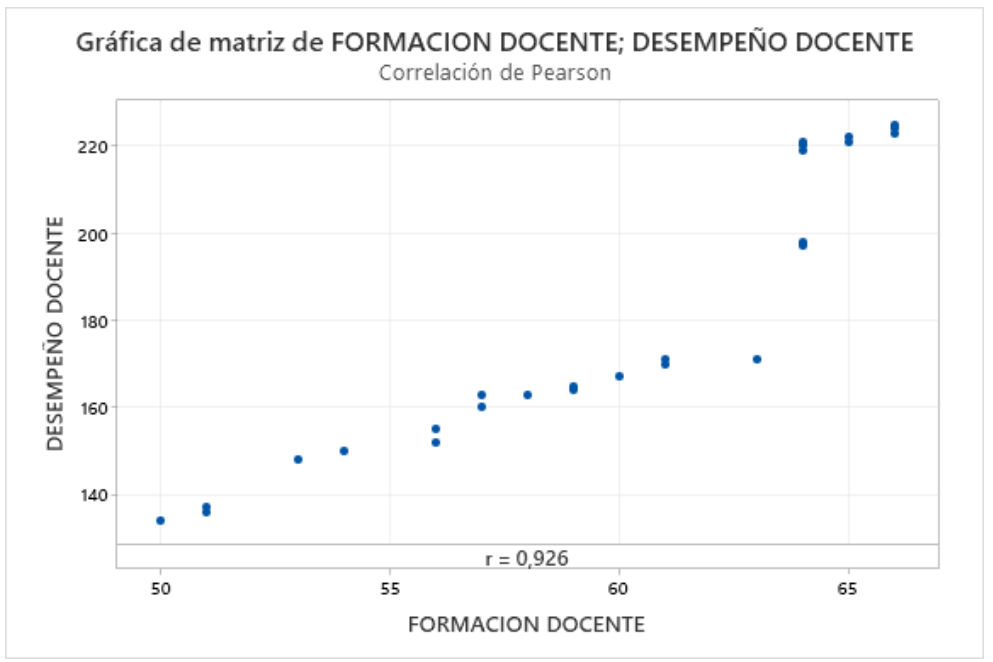

Fuente: Estudio en el contexto de la Unidad Educativa Provincia de Cotopaxi.

Nota: Datos obtenidos de la aplicación de la prueba de Spearman en Minitab.

La gráfica muestra la correlación entre la formación profesional docente y el desempeño laboral y como resultado nos ofreció un valor $r=0.926$, al contrastar este valor obtenido en la correlación de Pearson entre las dos variables podemos llegar a la conclusión de que existe un nivel de correlación muy alta entre los dos elementos. Por consiguiente, se puede afirmar que a mayor nivel de formación profesional docente mayor será el nivel del desempeño docente.

\section{Conclusiones}

- Mediante la aplicación del instrumento de autoevaluación de competencias docentes en los ciclos de formación y los puntajes que se obtuvieron al analizar de manera minuciosa se puede considerar que los docentes de la Unidad Educativa Provincia de Cotopaxi

- Presentan en su gran mayoría aún nivel excelente en relación con las 3 competencias desarrolladas para la presente investigación de tal modo que se concluye que si bien los docentes presentan un excelente auto concepto en relación con sus competencias como docentes es necesario no dejar a un lado las capacitaciones o las actividades extracurriculares que pueden mejorar el perfil dinamizador del docente y no permitir el conformismo con el conocimiento nuevo que requieren los docentes

- Por medio de la aplicación de la evaluación del desempeño docente por parte de 
los alumnos se lograron obtener datos relevantes e importantes para el análisis de la investigación, de tal modo que se puede concluir que los estudiantes se encuentran conformes con el desempeño laboral de los docentes. sin embargo, es necesario mejorar competencias en los docentes tales como el rol de acompañamiento del docente, manejo de los tics, atención a la diversidad y conocimiento a profundidad sobre las nuevas bibliografías de la asignatura que imparte el docente.

- Bajo una amplia gama de los análisis estadísticos que se realizaron en la investigación se puede concluir que la formación profesional docente es un elemento indispensable en el grado o calificación del desempeño laboral del profesorado de la unidad educativa provincia de Cotopaxi. por esta razón, se debe tomar cierta importancia en el perfil de formación de cada 1 de los docentes si se desea mejorar el nivel que desarrollan los docentes durante su jornada laboral. sin embargo, debe considerar oportuno el analizar ciertos factores externos que serán de vital importancia en el desarrollo del perfil de formación docente y por consiguiente en el desempeño laboral que refleje a sus estudiantes.

\section{Referencias Bibliográficas}

Amaya, T. S. (2016). saber pedagógico. Scielo, 6-13.

García, J.L. (2017). Obtenido de Desempeño docente: http://www.comie.org.mx/congreso/memoriaelectronica/v14/doc/2657.pdf

Gestión Educativa. (3 de marzo de 2016). https://gestioneducativa.educaweb.com/elnecesario-desarrollo-profesional-docente/

Mariana, S. M. (2019). CYBERTESIS. https://cybertesis.unmsm.edu.pe/handle/20.500.12672/10471

Martínez, M. F. (2018). Formación por competencia. Redalyc, 3-7. Mero, P. L. (2019). Rendimiento académico. Scielo, 2-7.

Ministerio de educación (2020). Sistema nacional de evaluación https://educacion.gob.ec/desempeno-del-docente-sne/

Ministerio de educación. (2020). Formación docente. https://educacion.gob.ec/formacion-docente/

Navarro, O. (30 de abril de 2018). https://ambitoeducativo.com/segun-el-infod-estasson-las-6-competencias-que-debera-tener-un-docente/

Ornelas, D. R. (17 de febrero de 2016). Slideshare. 
https://es.slideshare.net/danielrochaornelas/investigacin-documental-de-campo$y$ - experimental

Potosí, S. L. (2017). Desempeño docente. XIV:

http://www.comie.org.mx/congreso/memoriaelectronica/v14/doc/2657.pdf

Sampieri, H. (2018). Metodología de la investigación.

http://virtual.cuautitlan.unam.mx/rudics/wp-

content/uploads/2019/02/RUDICSv9n18p92_95.pdf

Suco, L. M. (2020). El desarrollo profesional docente. Illari, 1-3.

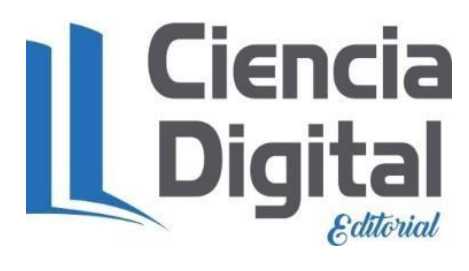


El artículo que se publica es de exclusiva responsabilidad de los autores y no necesariamente reflejan el pensamiento de la Revista Conciencia Digital.

\section{Ciencia}

El artículo queda en propiedad de la revista y, por tanto, su publicación parcial y/o total en otro medio tiene que ser autorizado por el director de la Revista Conciencia Digital.
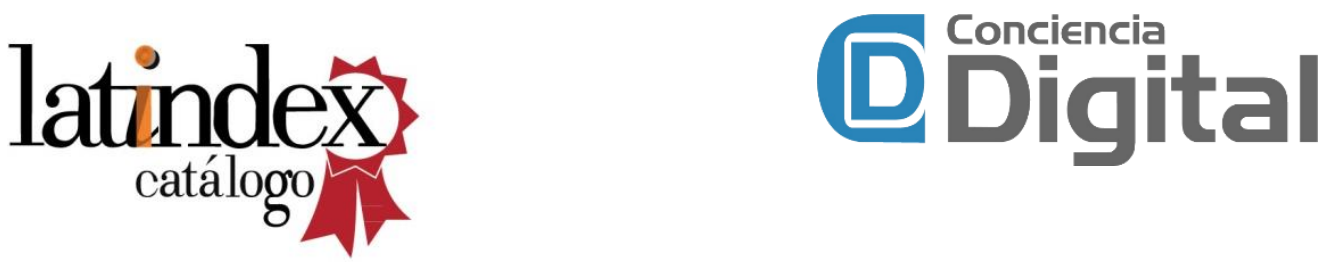

Indexaciones

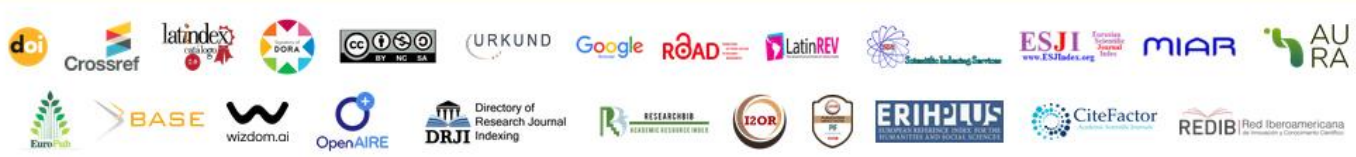

